\title{
Dynamics of the Transition from a Thin Accretion Disk to an Advection\#dominated Accretion Flow
}

\section{Citation}

Manmoto, Tadahiro, Shoji Kato, Kenji E. Nakamura, and Ramesh Narayan. 2000. "Dynamics of the Transition from a Thin Accretion Disk to an Advection\#dominated Accretion Flow." The Astrophysical Journal 529 (1): 127-37. https://doi.org/10.1086/308273.

\section{Permanent link}

http://nrs.harvard.edu/urn-3:HUL.InstRepos:41384924

\section{Terms of Use}

This article was downloaded from Harvard University's DASH repository, and is made available under the terms and conditions applicable to Other Posted Material, as set forth at http:// nrs.harvard.edu/urn-3:HUL.InstRepos:dash.current.terms-of-use\#LAA

\section{Share Your Story}

The Harvard community has made this article openly available.

Please share how this access benefits you. Submit a story.

Accessibility 
The ASTROPHYSICAL JOURNAL, 529:127-137, 2000 January 20

(c) 2000. The American Astronomical Society. All rights reserved. Printed in U.S.A.

\title{
DYNAMICS OF THE TRANSITION FROM A THIN ACCRETION DISK TO AN ADVECTION-DOMINATED ACCRETION FLOW
}

\author{
Tadahiro Manmoto, ${ }^{1}$ Shoji Kato, ${ }^{1,2}$ Kenji E. Nakamura, ${ }^{3}$ Ramesh Narayan ${ }^{1,4}$ \\ Received 1999 June 8; accepted 1999 September 7
}

\begin{abstract}
We consider an optically thin advection-dominated accretion flow (ADAF) that is connected at a finite transition radius to an outer optically thick, geometrically thin disk. We include turbulent energy transport and examine ADAF models that satisfy the following boundary conditions at the transition radius: (1) the temperature of the gas is much lower than the virial temperature, (2) the rotation is superKeplerian, and (3) the net radial flux of energy is outward. We numerically solve the height-integrated viscous hydrodynamic equations with these boundary conditions. We find that the Bernoulli parameter is positive for a wide range of radius, indicating that outflows may be possible from ADAFs. Turbulent energy transport enhances the Bernoulli parameter. We compare our numerical global solutions with two published analytical solutions. We find that the solution of Honma represents the transition region well, while the self-similar solution of Narayan \& Yi works better away from the transition. However, neither analytical solution is able to represent the density or angular momentum profile in the inner region of the ADAF, where the flow makes a sonic transition.
\end{abstract}

Subject headings: accretion, accretion disks - black hole physics - hydrodynamics — turbulence

\section{INTRODUCTION}

Three self-consistent and stable models of accretion flows are known (see Chen et al. 1995, who discuss these three models as well as a fourth unstable model by Shapiro, Lightman, \& Eardley 1976): (1) a geometrically thin, optically thick cool accretion disk model (hereafter, standard disk) (Shakura \& Sunyaev 1973), (2) a geometrically thick, optically thick advection-dominated accretion flow model (optically thick ADAF, also known as a slim disk) (Abramowicz et al. 1988), and (3) a geometrically thick, optically thin advection-dominated accretion flow model (optically thin ADAF) (Ichimaru 1977; Rees et al. 1982; Narayan \& Yi 1994 [hereafter NY], 1995a, 1995b; Abramowicz et al. 1995).

In a standard disk, the dissipated thermal energy is radiated efficiently and the disk is cool and geometrically thin. In an ADAF, on the other hand, the thermal energy is advected inward and the flow becomes very hot. In this paper our primary interest is in the optically thin, gaspressure-dominated branch of ADAFs, and we refer to them simply as ADAFs hereafter, but our equations and results are valid also for the radiation-pressure-dominated branch of ADAFs.

The ADAF model has been investigated extensively in recent years in a series of studies initiated by Narayan \& Yi (NY, 1995a, 1995b) and Abramowicz et al. (1995). The model has been used to explain the spectra of quiescent black hole X-ray binaries (e.g., Narayan, McClintock, \& Yi 1996; Narayan, Barret, \& McClintock 1997; Hameury et al. 1997); spectral transitions of X-ray binaries (Narayan 1996; Esin et al. 1998); the supermassive black hole source Sagittarius $\mathrm{A}^{*}$ at the center of our Galaxy (Narayan, Yi, \&

\footnotetext{
${ }^{1}$ Institute for Theoretical Physics, University of California, Santa Barbara, California 93106-4030.

${ }^{2}$ Nara Sangyo University, Sango, Ikoma-gun, Nara 630-8503, Japan.

${ }^{3}$ Department of Astronomy, Kyoto University, Sakyo-ku, Kyoto 6068502, Japan.

${ }^{4}$ Harvard-Smithsonian Center for Astrophysics, 60 Garden Street, Cambridge, MA 02138.
}

Mahadevan 1995; Manmoto, Mineshige, \& Kusunose 1997; Mahadevan 1998; Narayan et al. 1998); other lowluminosity nuclei of LINER galaxies, such as NGC 4258 (Lasota et al. 1996; Gammie, Narayan, \& Blandford 1999); and giant ellipticals such as M87, M60, and several others (Fabian \& Rees 1995; Reynolds et al. 1997; Mahadevan 1997; Di Matteo \& Fabian 1997; Di Matteo et al. 1999).

There have been a number of arguments to suggest that an accretion flow around a black hole may undergo a transition from a standard disk on the outside to an ADAF on the inside. The transition radius $R_{\mathrm{tr}}$ between the two zones has been estimated in some X-ray binaries by inferring the location of the innermost radius of the standard disk, either by fitting the soft component in the spectrum or from the width of the $\mathrm{H} \alpha$ line wings. The transition radius has also been estimated from studies of the hard component of black hole X-ray binary (BHXB) spectra. Narayan et al. (1996) and Narayan, Barret, \& McClintock (1997a) showed that the spectra of quiescent BHXBs are reproduced well by an ADAF model with a large transition radius, $R_{\mathrm{tr}} \sim$ $10^{3}-10^{4} R_{\mathrm{S}}$, where $R_{\mathrm{S}}$ is the Schwarzschild radius. In another study Manmoto et al. (1996) showed that the X-ray fluctuations that are seen in BHXBs could be explained as due to slowly growing instabilities in an ADAF. They used the timescale of individual X-ray shots to estimate the transition radius, assuming that the disturbances responsible for the X-ray shots are initiated near the transition between the $\mathrm{ADAF}$ and the standard disk.

There have been very few studies of the actual physical mechanism responsible for the transition from the outer standard disk to the inner ADAF. Nor do we discuss this topic here. We limit ourselves to a study of the requirements that an ADAF model must satisfy in order to be able to connect to an outer standard disk. Three such requirements have been identified in the literature.

First, we note that the physical state of the accreting gas in an ADAF away from the boundaries is described well by a self-similar solution derived by NY (see Narayan, Kato, \& Honma 1997b; Chen, Abramowicz, \& Lasota 1997). According to this solution, the temperature of the gas is 
close to the virial temperature and the radial velocity is comparable to $\alpha$ times the free-fall velocity, where $\alpha$ is the usual viscosity parameter. These features are a direct result of the domination of advective energy transport in the energy equation, which stabilizes these flows against the runaway thermal instability that usually affects optically thin accretion flows (Kato et al. 1997; Wu 1997). A major problem when constructing ADAF models with a transition is that the temperature and the radial velocity in an ADAF are orders of magnitude larger than those of a standard disk at the same radius. Therefore, if an ADAF is to be connected radially to a standard disk, the first requirement is that thermodynamic quantities like the temperature and dynamical quantities like the radial velocity must change by orders of magnitude in the transition region. This feature is in some sense realized in numerical global ADAF models in the literature where thin disklike outer boundary conditions are imposed (e.g., Narayan et al. 1997b; Manmoto et al. 1997; Nakamura et al. 1997).

The second requirement concerns the rotation of the flow. Abramowicz, Igumenshchev, \& Lasota (1998) noted that the accreting gas in an ADAF near the transition radius must rotate at a super-Keplerian angular velocity. This is because there is a pressure maximum in the flow whenever a standard disk is connected to an ADAF across a narrow transition layer. Narrow transitions are seen in numerical models (e.g., Narayan et al. 1997b).

The last and most important requirement is that an ADAF that is connected to a standard disk on the outside must have an outward energy flux (Kato \& Nakamura 1998). This is required for the following reason. In any steady standard disk model, the total energy flux passing through a cylindrical surface (whose axis is the rotation axis) is positive and (3/2)(GMM/R) at radius $R$, where $M$ is the mass of the central object and $\dot{M}$ is the mass accretion rate to the disk. Hence, an ADAF that is connected to a standard disk at $R_{\mathrm{tr}}$ must have a positive total energy flux larger than $(3 / 2)\left(G M \dot{M} / R_{\text {tr }}\right)$. Otherwise, the ADAF cannot connect thermally to the outer standard disk, since energy loss by radiation will inevitably occur at the transition region. The self-similar solution does not satisfy this requirement, since the total energy flux is zero (Kato \& Nakamura 1998). This does not mean that the self-similar solution is irrelevant, since some external heating may occur in real systems, for example, via a corona (Meyer \& Meyer-Hofmeister 1994). However, for the one-zone models we construct in this paper, such external energy sources are not possible, and therefore we must require the ADAF to have a net outward energy flux $\left[>(3 / 2)\left(G M \dot{M} / R_{\mathrm{tr}}\right)\right]$ at the outer boundary in order to match the thin disk.

We refer to the above three requirements as "connection conditions."

Honma (1996) was the first to construct models that satisfy the connection conditions. He assumed that when there is an entropy gradient in the radial direction in an ADAF, the turbulence in the flow would be a source of viscosity and at the same time would also transport energy (see also NY). Since an ADAF has entropy decreasing outward, the turbulent energy transport will carry energy outward, thereby enhancing the supply of energy to the outer disk.

In this paper, we consider ADAF models that connect to outer standard disks. We derive the general form of the outer boundary condition in an ADAF and construct global models that satisfy these boundary conditions. We investigate the effect of turbulent energy transport, a key feature of Honma's transition model, and we examine the differences between global ADAF models with energy transport and those without.

Honma (1996) also derived an analytical ADAF model that satisfies the connection conditions. The importance of this solution is, however, not widely appreciated and the nature of the solution has not been investigated in any detail. One of the goals of this paper is to explore the degree to which Honma's solution accurately represents real global solutions obtained by solving the equations numerically. To this end, we compare the numerical global solutions of the equations with Honma's solution as well as NY's selfsimilar solution. One of our aims is to understand the differences between Honma's and NY's solutions. We also extend Honma's solution to include the effect of radiative cooling.

The paper is organized as follows. In $\S 2$ we formulate the basic equations. In $\S 3$ we study global ADAF models that satisfy the connection conditions and examine the Bernoulli parameter of such global models. In $\S 4$ we explore the nature of Honma's analytical solution and clarify the regime of validity of the solution. We also clarify its relation to NY's self-similar solution. We conclude with a discussion in $\S 5$.

\section{BASIC EQUATIONS}

We consider a steady axisymmetric $\operatorname{ADAF}(\partial / \partial t=$ $\partial / \partial \phi=0)$. We define the vertically averaged pressure of the optically thin, gas-pressure-dominated gas as $p=\rho c_{\mathrm{s}}^{2}$, where $\rho$ is the vertically averaged density and $c_{\mathrm{s}}$ is the isothermal sound speed. (See Narayan \& Yi 1995a for a discussion of the interpretation of "vertical averaging" in the context of quasi-spherical ADAFs.) Assuming hydrostatic balance in the vertical direction, we write the pressure scale height as $H=c_{\mathrm{s}} / \Omega_{\mathrm{K}}$, where $\Omega_{\mathrm{K}}$ is the Keplerian angular velocity. Employing a simple dimensional argument, we write the surface density as $\Sigma=2 H \rho$ and the coefficient of (kinematic) shear viscosity as $v=\alpha c_{\mathrm{s}}^{2} / \Omega_{\mathrm{K}}$ (Shakura \& Sunyaev 1973), where $\alpha$ is the viscosity parameter.

With these definitions, the continuity equation, and the radial and azimuthal components of the momentum equation take the form

$$
\begin{gathered}
\dot{M}=-4 \pi R H \rho v \\
v \frac{d v}{d R}+\frac{1}{\rho} \frac{d}{d R}\left(\rho c_{\mathrm{s}}^{2}\right)=R\left(\Omega^{2}-\Omega_{\mathrm{K}}^{2}\right) \\
v \frac{d}{d R}\left(R^{2} \Omega\right)=\frac{1}{R H \rho} \frac{d}{d R}\left(\frac{\alpha \rho c_{\mathrm{s}}^{2} R^{3} H}{\Omega_{\mathrm{K}}} \frac{d \Omega}{d R}\right) .
\end{gathered}
$$

In equation (1), $\dot{M}$ is the mass accretion rate, which is defined to be positive for inflow, i.e., positive when the radial velocity $v$ is negative. In equations (2) and (3), $\Omega$ is the angular velocity of the gas.

The energy equation of the ADAF takes the form

$$
Q_{\mathrm{adv}}^{-}=Q_{\mathrm{vis}}^{+}-Q_{\mathrm{rad}}^{-}+Q_{\mathrm{turb}}^{+} \text {. }
$$

This equation gives the balance of the heating rate and cooling rate per unit surface area. The viscous heating rate, $Q_{\text {vis }}^{+}$, is given by

$$
Q_{\mathrm{vis}}^{+}=\frac{2 \alpha \rho c_{\mathrm{s}}^{2} R^{2} H}{\Omega_{\mathrm{K}}}\left(\frac{d \Omega}{d R}\right)^{2} .
$$


As in NY, we introduce a parameter $f$ that describes the importance of radiative cooling $Q_{\mathrm{rad}}^{-}$,

$$
Q_{\mathrm{vis}}^{+}-Q_{\mathrm{rad}}^{-}=f Q_{\mathrm{vis}}^{+} \text {. }
$$

The left-hand side of equation (4) is the advective cooling:

$$
Q_{\mathrm{adv}}^{-}=\Sigma v T \frac{d s}{d R}=3(1+\epsilon) \rho H v \frac{d c_{\mathrm{s}}^{2}}{d R}-2 c_{\mathrm{s}}^{2} H v \frac{d \rho}{d R},
$$

where $T$ is the temperature, $s$ is the entropy, and $\epsilon$ is a parameter that is related to the ratio of specific heats $\gamma$ (NY): $\epsilon=(5 / 3-\gamma) /(\gamma-1)$. We have $\epsilon=0$ when $\gamma=5 / 3$ and $\epsilon=1$ when $\gamma=4 / 3$. The last term on the right-hand side of equation (4) is the heating due to turbulent energy transport. This term was originally considered in the context of convective energy transport by NY, and later played an important role in Honma's (1996) model of the transition. Following Honma, we write $Q_{\text {turb }}^{+}$as

$$
Q_{\mathrm{turb}}^{+}=-\frac{1}{R} \frac{d}{d R}\left(2 R H F_{\mathrm{turb}}\right),
$$

where $F_{\text {turb }}$ is the vertically averaged energy flux due to turbulence:

$$
F_{\mathrm{turb}}=-\rho K_{T} T \frac{d s}{d R}=-3(1+\epsilon) \frac{\alpha_{T} \rho c_{\mathrm{s}}^{2}}{\Omega_{\mathrm{K}}} \frac{d c_{\mathrm{s}}^{2}}{d R}+\frac{2 \alpha_{T} c_{\mathrm{s}}^{4}}{\Omega_{\mathrm{K}}} \frac{d \rho}{d R} .
$$

We have introduced a diffusion constant, $K_{T}$, associated with the turbulent energy transport and have written it as $K_{T}=\alpha_{T} c_{\mathrm{s}}^{2} / \Omega_{\mathrm{K}}$ with a dimensionless parameter $\alpha_{T}$. This is done purely on dimensional grounds, in analogy with the Shakura-Sunyaev model of viscosity. The parameter $\alpha_{T}$ introduced here is identical to the convective transport coefficient $\alpha_{c}$ in NY.

\section{GLOBAL SOLUTIONS}

We compute global ADAF solutions by numerically integrating equations (1)-(4) with proper boundary conditions that satisfy the connection conditions. Let $R_{\mathrm{tr}}$ be the transition radius (i.e., the outer boundary of the ADAF), and let us define $x=R / R_{\mathrm{tr}}$. Since the temperature at the boundary is much lower than the virial temperature, we set $c_{\mathrm{s}}^{2}=0$ at $x=1$. The condition that the flow is super-Keplerian at $x=1$ requires that the term $(1 / \rho) d\left(\rho c_{\mathrm{s}}^{2}\right) / d R$ must have a finite positive value (see eq. [2]). These two conditions lead to

$$
\begin{gathered}
c_{\mathrm{s}}^{2}=c_{0}^{2}(1-x)+O(1-x)^{2}, \\
\rho=\rho_{0}(1-x)^{-n}+O(1-x)^{-n+1},
\end{gathered}
$$

where $c_{0}$ and $\rho_{0}$ are constants and $n$ is positive. We see that the boundary at $x=1$ is a singular point where the density diverges. The value of $n$ is easily seen to be $n=3 / 2$ from the requirement that a finite amount of angular momentum should be transported outward at $x=1$. To determine $c_{0}^{2}$ and $\rho_{0}$, we expand all physical quantities around $x=1$ as power series in $(1-x)$, and equate terms of the same power in the basic equations. This procedure is given in the
Appendix. In the case of $\alpha_{T}=0$, for example, we find

$$
\frac{c_{\mathrm{s}}^{2}}{v_{\mathrm{K}}^{2}}=\frac{2 f}{3(\epsilon+2) g-f}(1-x)+O(1-x)^{2},
$$

$\frac{\Omega^{2}}{\Omega_{\mathrm{K}}^{2}}=\frac{3(\epsilon+2) g}{3(\epsilon+2) g-f}$

$$
\begin{array}{r}
\times\left[1-\left(3-\frac{2}{g}\right)(1-x)+O(1-x)^{2}\right], \\
\frac{v}{v_{\mathrm{K}}}=-\frac{2 \alpha f^{2}}{g[3(\epsilon+2) g-f]}(1-x)+O(1-x)^{2},
\end{array}
$$

where

$$
g=-\left(\frac{d \ln \Omega}{d \ln R}\right)_{R=R_{\mathrm{tr}}}^{-1}
$$

is a free parameter that is given uniquely once the global solution is obtained and $\Omega_{\mathrm{K}}(R)=\left(G M / R^{3}\right)^{1 / 2}$ and $v_{\mathrm{K}}=r \Omega_{\mathrm{K}}$ are the Keplerian angular velocity and the Keplerian azimuthal velocity in the Newtonian potential. We see from equation (13) that we have a super-Keplerian rotation at $r=R_{\mathrm{tr}}$ for an ADAF with $g>0$ (which is usually the case) and $f=1$.

The vertically integrated total energy flux $W$ through the boundary is given by (see the Appendix):

$$
W=\frac{G M \dot{M}}{R_{\mathrm{tr}}} \frac{3(\epsilon+2) g-f}{2(\epsilon+2) g-f},
$$

where $M$ is the mass of the central black hole. The energy flux $W$ is positive and larger than $(3 / 2)\left(G M \dot{M} / R_{\text {tr }}\right)$ when

$$
g>\frac{f}{2(\epsilon+2)},
$$

which holds as long as $g>\frac{1}{4}$, which is usually satisfied for ADAFs. Thus the above transition zone solution gives general outer boundary conditions that are consistent with the connection conditions.

Because of the singularity at the boundary, we terminate the numerical solutions at $x=0.99$ and apply the boundary conditions (12)- (14) at that point. We also impose a notorque condition $(d \Omega / d R=0)$ and an adiabatic condition $(d s / d R=0)$ at the inner boundary at $R=R_{\mathrm{S}}$, where $R_{\mathrm{S}}$ is the Schwarzschild radius. We adjust the specific angular momentum swallowed by the black hole $l_{\text {in }}$ using a relaxation scheme such that the solution satisfies the standard regularity conditions at the sonic point (e.g., Honma 1996; Narayan et al. 1997b). The no torque condition has no effect on the outer boundary. To simulate relativistic effects near the central black hole, we use Paczyński \& Wiita's (1980) pseudo-Newtonian potential and set $\Omega_{\mathrm{K}}(R) /\left(G M / R^{3}\right)^{1 / 2}=R /\left(R-R_{\mathrm{S}}\right)$.

\subsection{Parameter Dependences of Global Solutions}

Figure 1 shows the $\alpha$-dependence of the numerical global solutions. The results are similar to those obtained by others, e.g., Narayan et al. (1997b) and Chen et al. (1997). The sound speed $c_{s}$ is essentially independent of $\alpha$, while the specific angular momentum $l=\Omega R^{2}$ and the density $\rho$ decrease with increasing $\alpha$. Near the outer boundary, however, both $c_{\mathrm{s}}$ and $l$ are independent of $\alpha$, while $\rho$ is sensitive to the value of $\alpha$. This is due to the fact that $c_{\mathrm{s}}$ and $l$ in the transition zone solution (12)-(14) given in the pre- 

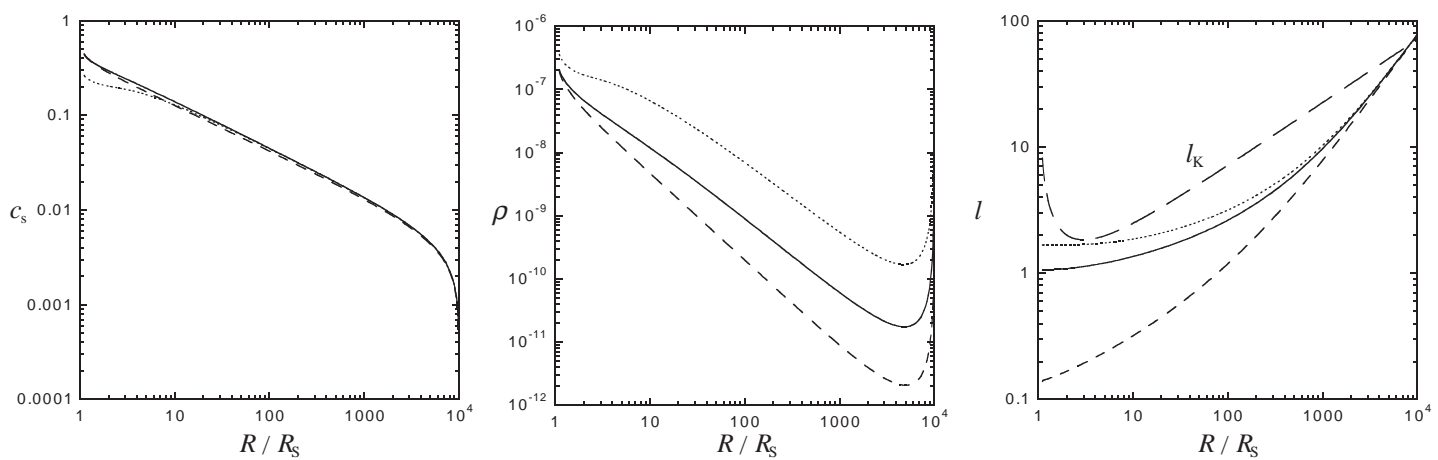

Fig. 1.-Numerical global solutions for $\epsilon=0, r_{\mathrm{tr}}=10^{4} R_{\mathrm{S}}$, and three values of $\alpha$. Left panel: Isothermal sound speed $c_{\mathrm{s}}$. Center panel: Density $\rho$. Right panel: Specific angular momentum $l$. Solid lines correspond to $\alpha=0.1$, dashed lines to $\alpha=1.0$, and dotted lines to $\alpha=0.01$. The long-dashed line in the right panel represents the Keplerian angular momentum. All quantities shown here are independent of the mass of the central object and the mass accretion rate, except for density. The density depends on the mass accretion rate as $\rho \propto \dot{m}$, where $\dot{m}=\dot{M} / \dot{M}_{\mathrm{Edd}}$ and $\dot{M}_{\mathrm{Edd}}$ is the Eddington mass accretion rate (defined with an efficiency factor of 0.1 ). In this and subsequent figures, the density corresponds to $\dot{m}=0.01$.

vious section do not depend explicitly on $\alpha$, while $v$ does, and this affects $\rho$ through the continuity equation.

Figure 2 shows the $\epsilon$-dependence of the global solution (solid curves). We consider two extreme values of $\epsilon$ in this paper: $\epsilon=0$, which corresponds to $\gamma=5 / 3$, and $\epsilon=4 / 3$, which corresponds to $\gamma=4 / 3$. When $\epsilon$ is changed from zero to unity, keeping other physical parameters fixed, $c_{\mathrm{s}}$ decreases and $\rho$ increases. This is again obvious from the transition solution (12)-(14). Figure 2 also shows that the effect of turbulent energy transport on the global solution is small. Interestingly, the solution corresponding to $\alpha_{T}=\alpha$, $\epsilon=1$ asymptotically approaches that for $\alpha_{T}=0, \epsilon=0$ near the outer boundary. This is consistent with the expectations of Honma's analytical solution as discussed in $\S 4$.

\subsection{Bernoulli Parameter}

Narayan \& Yi (1994) showed that the Bernoulli parameter $b$ (三Bernoulli constant $\left./ v_{K}^{2}\right)$ in their self-similar solution is given by

$$
\begin{aligned}
b & =\frac{1}{v_{\mathrm{K}}^{2}}\left(\frac{1}{2} v^{2}+\frac{1}{2} \Omega^{2} R^{2}-\psi+\frac{\gamma}{\gamma-1} c_{\mathrm{s}}^{2}\right) \\
& =-\frac{\Omega^{2} R^{2}}{2 v_{\mathrm{K}}^{2}}+\left(\frac{\gamma}{\gamma-1}-\frac{5}{2}\right) \frac{c_{\mathrm{s}}^{2}}{v_{\mathrm{K}}^{2}} \approx \frac{3 \epsilon-\epsilon^{\prime}}{5+2 \epsilon^{\prime}},
\end{aligned}
$$

where $\psi$ is the gravitational potential energy, $\epsilon^{\prime}=\epsilon / f$, and the last relation is for the approximation of $\alpha \ll 1$. It is seen that $b$ is zero for $\epsilon=0$ and is positive for $\epsilon>0$ and $f>\frac{1}{3}$.

In the absence of viscous and turbulent energy transport, the Bernoulli constant, $b v_{\mathrm{K}}^{2}$, is a conserved quantity. Thus if the accreting gas has a positive Bernoulli parameter at a certain radius, and if some physical process allows the gas to flow outward, both adiabatically and without viscous stresses, then the gas will be able to reach infinity with positive energy (Narayan \& Yi 1994, 1995a). As Abramowicz, Lasota, \& Igmenshchev (1999) have pointed out (see also Narayan \& Yi 1994), however, the Bernoulli constant must be negative at the event horizon when proper boundary conditions are imposed. In addition, the Bernoulli constant in a standard thin disk is always negative. Hence, in ADAF models in which the gas makes a transition from a thin disk on the outside and falls into a black hole on the inside, the Bernoulli constant is negative at both boundaries.

Nakamura (1998) showed, using Honma's analytical solution, that the Bernoulli constant for $\epsilon=0$ is negative except for the innermost region of ADAFs. As we will show later, however, Honma's solution is not a good approximation to the inner regions of an ADAF. This motivates us to consider the Bernoulli parameter in the numerical global solutions. The positivity of the Bernoulli constant is due to energy transport from the inner to outer regions of the flow. Some energy transport occurs via viscosity, but we expect that turbulence will enhance the outward energy transport and make the Bernoulli parameter larger (even though the
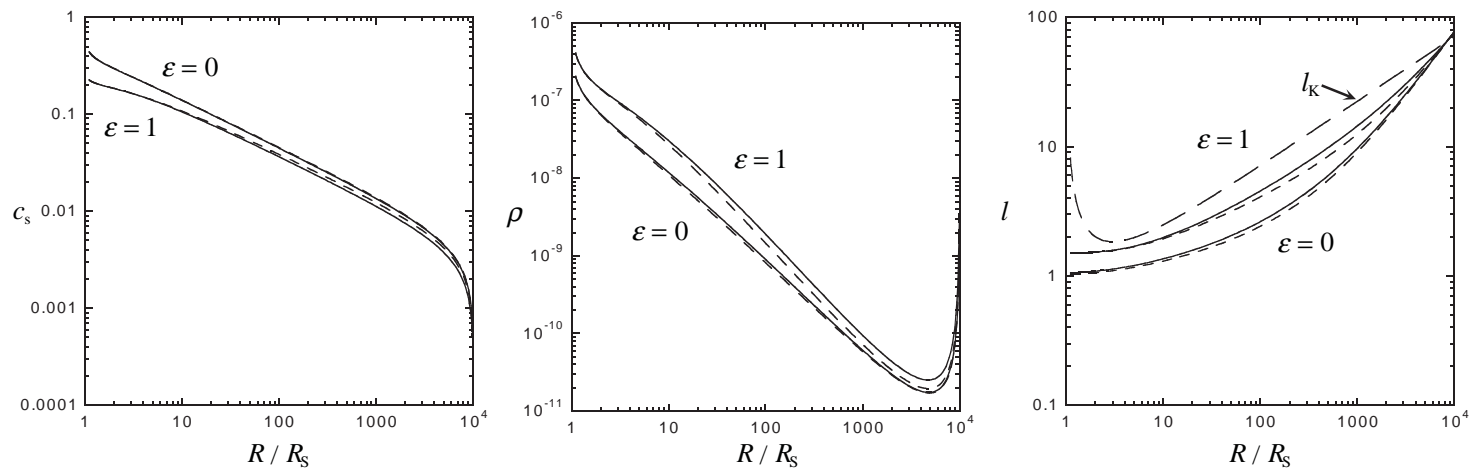

Fig. 2.-Numerical global solutions for two values of $\epsilon, 0$ and 1 , and two values of $\alpha_{T}: \alpha_{T}=0$ (solid lines) and $\alpha_{T}=\alpha$ (dashed lines). The models correspond to $\alpha=0.1$ and $R_{\mathrm{tr}}=10^{4} R_{\mathrm{S}}$. Left panel: Isothermal sound speed $c_{\mathrm{s}}$. Center panel: Density $\rho$ (for $\dot{m}=0.01$ ). Right panel: Specific angular momentum $l$. The long-dashed line in the right panel represents the Keplerian angular momentum. 
inclusion of a turbulent $\alpha_{T}$ does not modify the structure of the flow very much; see Fig. 2). We investigate this question numerically.

Figure 3 shows the Bernoulli parameter of global solutions with various values of $R_{\mathrm{tr}}$. Also plotted are the constant values calculated from NY's self-similar solution. We notice that, even though the Bernoulli parameter is negative at the outer boundary and zero at the horizon, it is positive over a wide range of radius in the interior, especially when $R_{\mathrm{tr}}$ is large. (Note that the Bernoulli parameter $b$ is proportional to $\left(R / R_{\mathrm{S}}-1\right)$ because it is normalized by $v_{\mathrm{K}}^{2}$. In our calculations, the Bernoulli constant $b v_{\mathrm{K}}^{2}$ at the horizon is actually negative and even less than that at the outer boundary, as demanded by the no torque condition at the horizon; see Abramowicz et al. 1999.) Even in the case of $\epsilon=0$, where NY's self-similar solution gives $b=0$ and Honma's analytical solution gives $b<0$ everywhere except for a localized innermost region (Nakamura 1998), the numerical models give a positive $b$ over a fairly wide range of radius. In the case of $\epsilon=1, \alpha_{T}=0$, and $R_{\mathrm{tr}}$ large (e.g., $10^{4} R_{\mathrm{S}}$ ), the maximum value of $b$ in the numerical solution approaches the analytical value given by NY.

A point worth emphasizing is that energy transport due to turbulence enhances the value of $b$ in the case of $\epsilon=1$ and large $R_{\mathrm{tr}}$. The case of $\epsilon \sim 1$ is really expected because of the frozen-in turbulent magnetic field (Narayan \& Yi 1995b). In such a case we can perhaps expect further $\alpha_{T}=\alpha$. We also found that even when the viscosity is small $(\alpha=0.01)$, results are qualitatively the same as in the case of $\alpha=0.1$, although the maximum value of $b$ is $\sim 0.1$ in the case of $\epsilon=1$, which is different from the results given in Abramowicz et al. (1999).

We conclude that the outflow might be expected in an ADAF, as suggested by Blandford \& Begelman (1998), because a necessary condition for outflows $(b>0)$ is satisfied in the wide range of radius even when $\epsilon$ is close to zero and the viscosity is small. One should note, however, that positivity of $b$ by itself is not the sufficient condition for outflows; there are additional two requirements, which are not easy to arrange: (1) the streamlines of the inflowing gas are somehow directed outward (NY) and (2) the out- flowing gas is able to escape infinity adiabatically and inviscidly keeping positivity of $b$. We should comment as to the first requirement. It is unlikely that ADAFs can produce outflows of purely hydrodynamical origin, considering that the several two-dimensional time-dependent numerical models (e.g., Abramowicz et al. 1999) show that all matter enters the black hole, yet $b$ is positive, as is claimed by Abramowicz et al. (1999). We further repeat the one of the statements in Abramowicz et al. (1999): the outflow from an ADAF might occur as a result of nonhydrodynamical factors such as large-scale global magnetic fields and radiation, which cannot be modeled in the value of $\epsilon$. In any case, one of necessary conditions for outflow is cleared by positivity of $b$ in the wide region, but more considerations are necessary to judge theoretically whether outflows from ADAFs are really expected or not.

\section{ANALYTICAL SOLUTIONS}

\subsection{Honma's Solution}

Neglecting the inertial term $v d v / d R$ in equation (2), Honma (1996) found an analytical solution that satisfies the connection conditions. In terms of $x$ as defined in this paper, Honma's solution takes the form

$$
\begin{gathered}
v=-\alpha \frac{2}{5}(1-x) v_{\mathrm{K}}(R), \\
\Omega=\sqrt{\frac{6}{5}} x^{1 / 2} \Omega_{\mathrm{K}}(R), \\
c_{\mathrm{s}}=\left[\frac{2}{5}(1-x)\right]^{1 / 2} v_{\mathrm{K}}(R) .
\end{gathered}
$$

Near the outer boundary $(x \sim 1)$, the temperature decreases sharply and the rotation becomes super-Keplerian, as required by the connection conditions.

It is easy to show that Honma's solution satisfies equations (1)-(3), provided the inertial term can be neglected. The condition under which the solution satisfies equation (4) is found to be

$$
\left(1-\frac{\alpha_{T}}{\alpha}\right) \epsilon+2(1-f) x=0 .
$$

Thus the solution is valid if $f=1$ and either one of two conditions is satisfied: (1) $\epsilon=0$ (Kato \& Nakamura 1998)
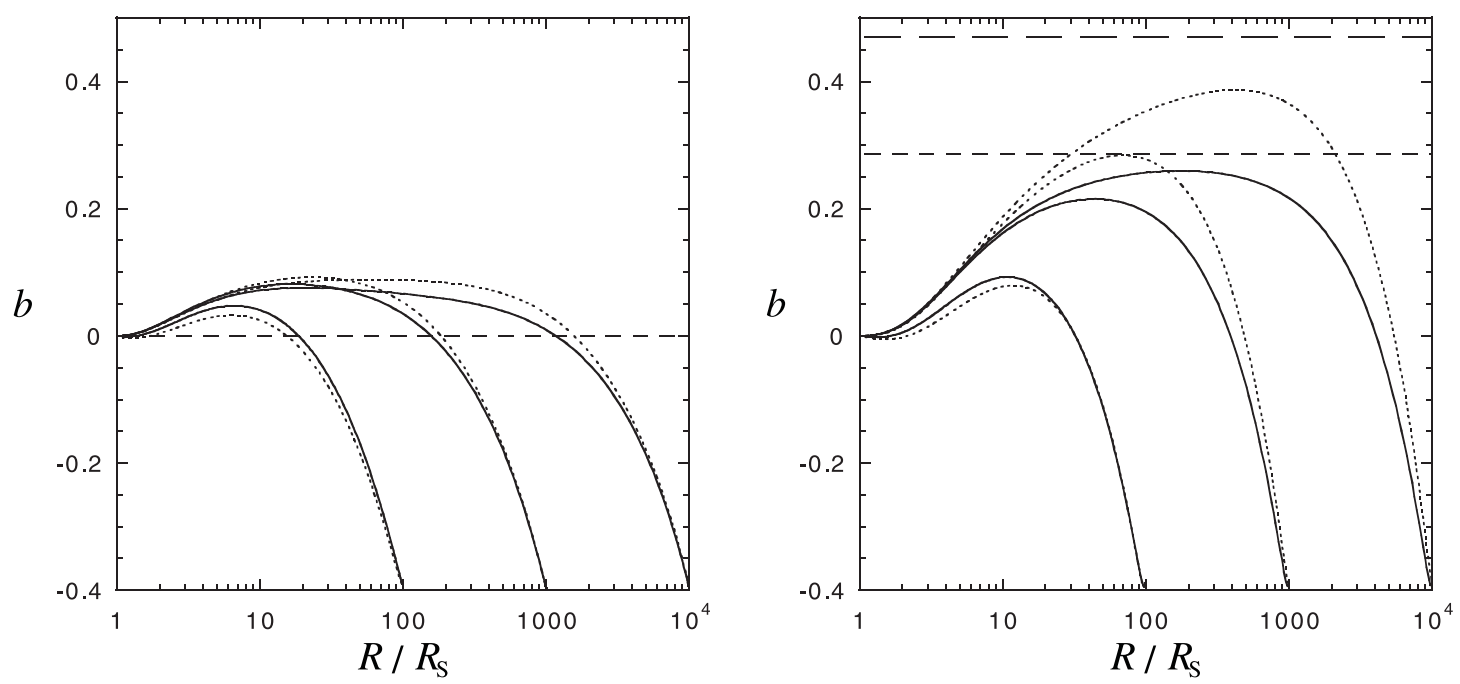

FIG. 3.-Bernoulli parameter $b$ as a function of radius. Left panel: Models with $\epsilon=0$. Right panel: Models with $\epsilon=1$. Global solutions with $R_{\mathrm{tr}}=100 R_{\mathrm{S}}$, $R_{\mathrm{tr}}=1000 R_{\mathrm{S}}, R_{\mathrm{tr}}=10^{4} R_{\mathrm{S}}$ are shown. Solid lines correspond to models with $\alpha_{T}=0$ and dotted lines to models with $\alpha_{T}=\alpha$. The dashed lines and the long-dashed line represent the values of $b$ predicted by NY's self-similar solution with $\alpha_{T}=0$ and $\alpha_{T}=\alpha$, respectively. 
or (2) $\alpha_{T}=\alpha$ (Honma 1996). Even when equation (22) is not satisfied, the solution is still found to be a good approximation to the flow around the transition zone $(x \sim 1)$, as we show in $\S 4.3$.

Because Honma's solution neglects the inertial term, it is invalid for small $x$, where $v$ is no longer negligible compared to $c_{\mathrm{s}}$.

\subsection{The Self-Similar Solution}

In this subsection we give an extended form of NY's selfsimilar solution, taking into account the effect of turbulent energy transport. Assuming $\epsilon^{\prime}$ to be constant and all the physical variables with the dimensions of velocity to be proportional to the Keplerian velocity $v_{\mathrm{K}}(R)=(G M / R)^{1 / 2}$, we have the following solution:

$$
\begin{gathered}
v=-\left(5+2 \epsilon^{\prime}-\frac{4}{3} \frac{\alpha_{T}}{\alpha} \epsilon^{\prime}\right) \frac{g\left(\alpha, \epsilon^{\prime}\right)}{3 \alpha} v_{\mathrm{K}}(R), \\
\Omega=\left\{\frac{2 \epsilon^{\prime}\left[5+2 \epsilon^{\prime}-(4 / 3)\left(\alpha_{T} / \alpha\right) \epsilon^{\prime}\right]\left[1-(2 / 3)\left(\alpha_{T} / \alpha\right)\right] g\left(\alpha, \epsilon^{\prime}\right)}{9 \alpha^{2}}\right\}^{1 / 2} \\
\times \Omega_{\mathrm{K}}(R), \\
c_{\mathrm{s}}=\left\{\frac{2\left[5+2 \epsilon^{\prime}-(4 / 3)\left(\alpha_{T} / \alpha\right) \epsilon^{\prime}\right]}{9} \frac{g\left(\alpha, \epsilon^{\prime}\right)}{\alpha^{2}}\right\}^{1 / 2} v_{\mathrm{K}}(R), \\
g\left(\alpha, \epsilon^{\prime}\right) \equiv\left\{1+\frac{18 \alpha^{2}}{\left[5+2 \epsilon^{\prime}-(4 / 3)\left(\alpha_{T} / \alpha\right) \epsilon^{\prime}\right]^{2}}\right\}^{1 / 2}-1
\end{gathered}
$$

This reduces to the original self-similar solution of Narayan \& Yi (1994) in the limit of $\alpha_{T}=0$.

Let us compare the self-similar solution to Honma's solution. First, the self-similar solution has no outer boundary, while Honma's solution does. Second, the self-similar solution naturally reduces to a standard disk in the limit of very efficient cooling, $f \rightarrow 0$ and $\epsilon^{\prime} \rightarrow \infty$, while Honma's solution satisfies the equations only for $f=1$ (fully advectiondominated flow). The self-similar solution can connect to an outer standard disk, as far as the temperature is concerned, by moving $f$ to 0 by hand as a function of radius, but the rotation is always sub-Keplerian and the energy flux outward is always zero, which contradicts the other two connection conditions. Third, when $\epsilon=0$, the self-similar solution gives a Bondi-type accretion flow with no rotation, while Honma's solution gives a rotating flow. Fourth, when we include turbulent energy transport, the self-similar solution gives a nonrotating flow for $\alpha_{T}=3 / 2 \alpha$, whereas Honma's solution rotates for all values of $\alpha_{T}$. (Note that we expect $\alpha_{T} \lesssim \alpha$ in general, so this difference is not of practical consequence.)

\subsection{Comparison of Analytical and Global Solutions}

In this subsection we compare the two analytical solutions described above with the global solutions obtained numerically in the previous section.

Consider first the case of $\epsilon=0$. In this case, Honma's solution satisfies the equations for any value of $\alpha_{T}$, so long as the inertial term in equation (2) may be neglected. Moreover, the solution is independent of the value of $\alpha_{T}$, which can be understood by considering the energy flux due to turbulent transport (eq. [9]):

$$
4 \pi R H F_{\text {turb }} \propto \alpha_{T}\left(2+\frac{\epsilon}{x}\right) \text {. }
$$

We see that, when $\epsilon=0,4 \pi R H F_{\text {turb }}$ is a constant independent of radius. Therefore, even though the magnitude of the energy flux does depend on $\alpha_{T}$, nevertheless, the turbulent heating term $Q_{\text {turb }}^{+}$is zero because the divergence of the flux vanishes. Of course, in the full global solutions with proper boundary conditions, $F_{\text {turb }}$ must be zero at the event horizon (where the flow is adiabatic) and hence $Q_{\text {turb }}^{+}$must be negative in the innermost region of the flow. This effect is not reproduced by the analytical solution.

Figure 4 compares Honma's analytical solution with the global solution for $\epsilon=0$ and $\alpha_{T}=0$. The outer boundary is set at $R_{\text {tr }}=10^{4} R_{\mathrm{s}}$. Notice that $c_{\mathrm{s}}$ is very nicely fitted by Honma's solution at all radii (left panel). However, the solution does not fit the profiles of the density or the specific angular momentum in the inner region of the flow. The discrepancy is a factor of $\sim 10$ for the density and $\sim 100$ for the angular momentum. Surprisingly, the fit is poor even in an intermediate region, $r \sim 100-1000 R_{\mathrm{S}}$, where the inertial term is negligible. The solution does accurately describe the structure of the outer boundary layer where the density increases sharply and the rotation becomes superKeplerian.

Figure 5 shows the effect of moving the outer boundary to $r=100 R_{\mathrm{s}}$. The sound speed $c_{\mathrm{s}}$ is still fitted well by Honma's analytical solution for a wide range of radius, but the region where the solution gives a good fit to $\rho$ and $l$
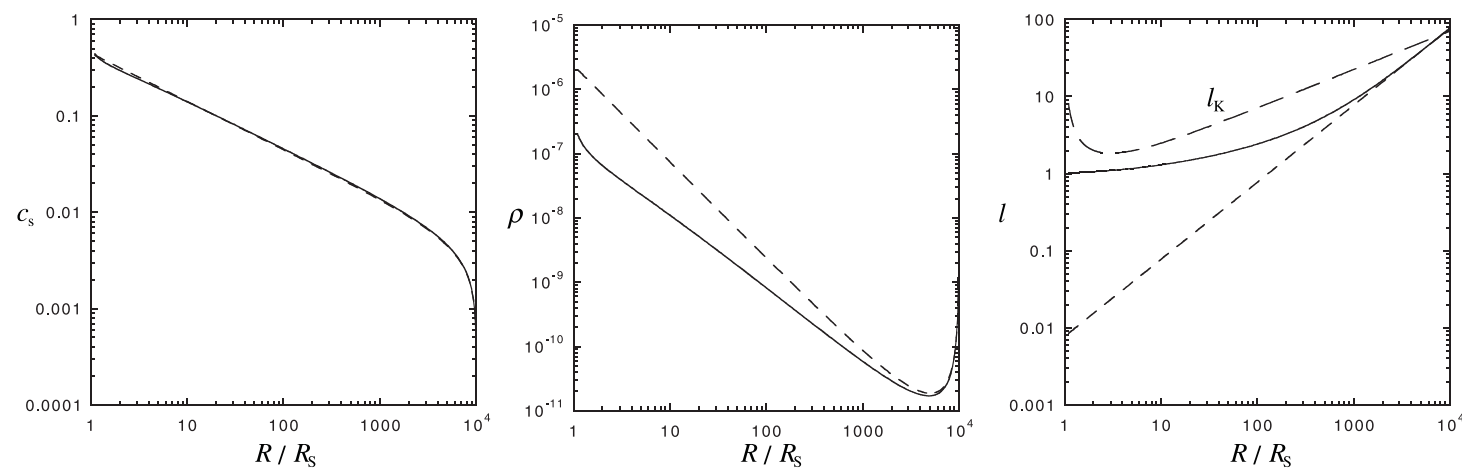

FIG. 4.-Comparison of numerical global solutions with Honma's analytical solution for $\epsilon=0, \alpha=0.1, \alpha_{T}=0, R_{\mathrm{tr}}=10^{4} R_{\mathrm{S}}$. Left panel: Isothermal sound speed $c_{\mathrm{s}}$. Center panel: Density $\rho$ (for $\dot{m}=0.01$ ). Right panel: Specific angular momentum $l$. Solid lines represent the numerical solutions and dashed lines show Honma's solution. The long-dashed line in the right panel represents the Keplerian angular momentum. 

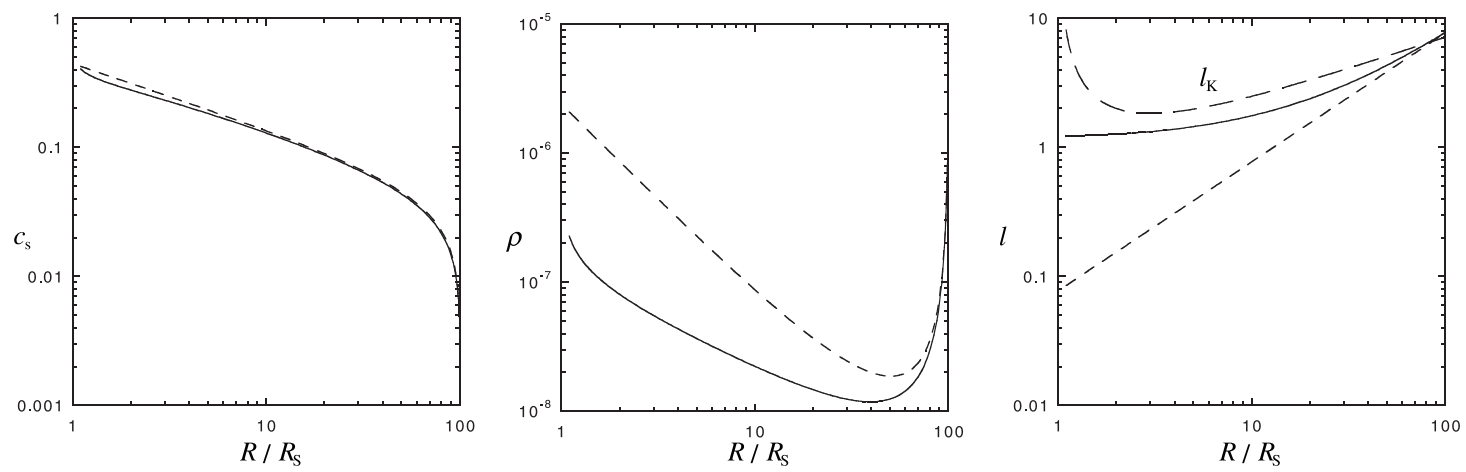

FIG. 5. - Similar to Fig. 4, but for $R_{\mathrm{tr}}=100 R_{\mathrm{S}}$

becomes narrower. As in the previous case, the region near the transition radius is represented well.

Now, let us proceed to the case of $\epsilon=1$. Figure 6 compares Honma's analytical solution and NY's self-similar solution with the numerical global solution for $\alpha_{T}=0$. We see that in the region near the outer boundary, Honma's solution once again accurately traces the global solution, while NY's self-similar solution does not represent the structure of this zone. In the intermediate zone, however, NY's self-similar solution gives a better fit to the profiles of $c_{\mathrm{s}}$ and $l$. In this sense, the two solutions are complementary. NY's self-similar solution gives a good approximation in the intermediate zone (as already noted by Narayan et al. 1997b), while Honma's solution represents well the structure of the transition region.
Figure 7 shows a solution with $\epsilon=1$ and a smaller $r_{\mathrm{tr}}=$ $100 R_{\mathrm{S}}$. Now we see that both analytical solutions give poor fits to the global solutions. Only Honma's solution barely fits the outermost part of the flow correctly. Thus we conclude that when the transition radius is as small as $R_{\mathrm{tr}}=$ $100 R_{\mathrm{S}}$, we have no analytical solution that can represent the inner parts of the flow, especially the density and angular momentum. This is due to the fact that in both Honma's and NY's analytical solutions the transonic nature of the flow near the black hole is not taken into account.

\subsection{Effect of Cooling}

We extend Honma's solution, which only treats fully advection-dominated flows $(f=1)$, to the case of ADAFs with partial cooling $(f<1)$. Introducing a new parameter $a$,
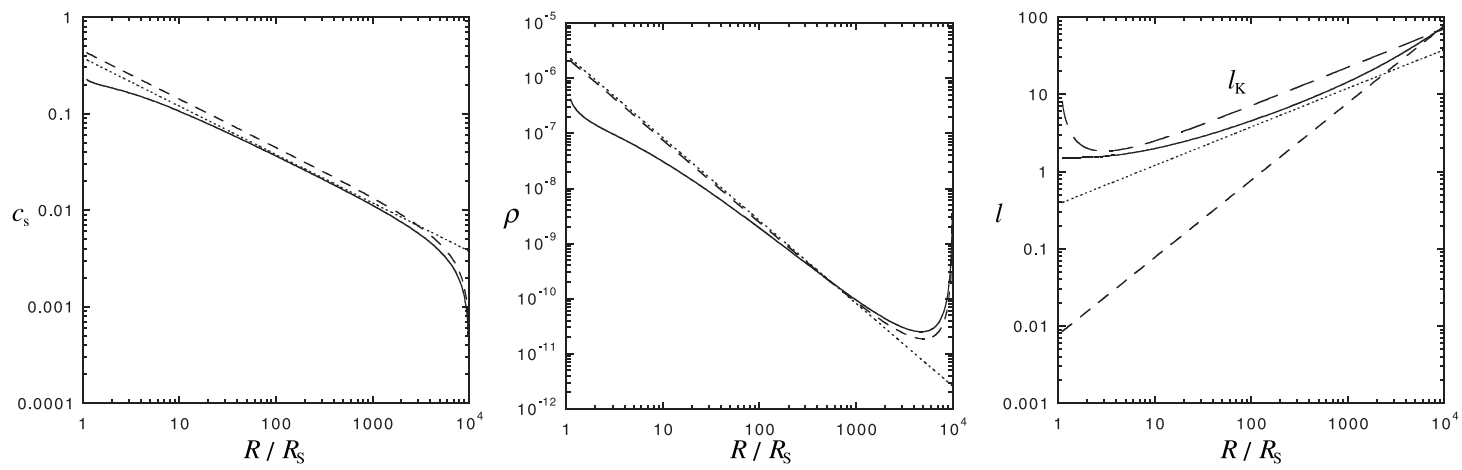

Fig. 6.-Comparison of numerical global solutions with Honma's and NY's analytical solutions for $\epsilon=1, \alpha=0.1, \alpha_{T}=0, R_{\mathrm{tr}}=10^{4} R_{\mathrm{S}}$. Left panel: Isothermal sound speed $c_{\mathrm{s}}$. Center panel: Density $\rho$ (for $\dot{m}=0.01$ ). Right panel: Specific angular momentum $l$. Solid lines correspond to the numerical solutions, dashed lines to Honma's analytical solution and dotted lines to NY's analytical self-similar solution. The long-dashed line in the right panel represents the Keplerian angular momentum.
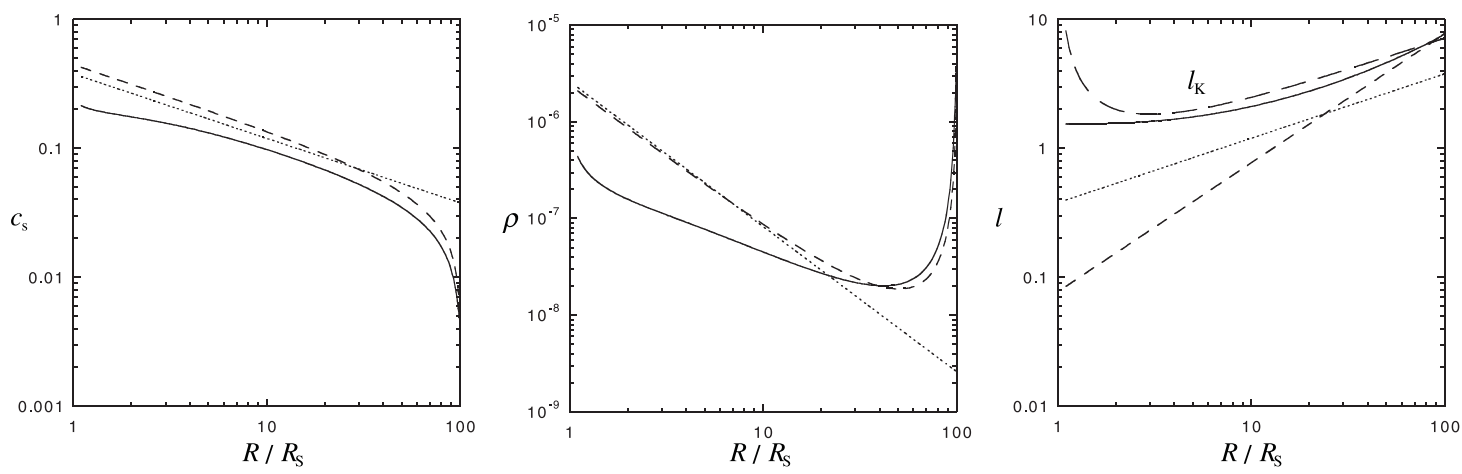

FIG. 7.- Similar to Fig. 6 , but for $R_{\mathrm{tr}}=100 R_{\mathrm{S}}$ 
which we show below to be related to the value of $f$, we consider the following extended form of Honma's solution:

$$
\begin{gathered}
v=-\alpha \frac{(3-a)}{5}\left(1-x^{a}\right) v_{\mathrm{K}}(R), \\
\Omega=\sqrt{\frac{a+5}{5}} x^{a / 2} \Omega_{\mathrm{K}}(R), \\
c_{\mathrm{s}}=\sqrt{\frac{2}{5}}\left(1-x^{a}\right)^{1 / 2} v_{\mathrm{K}}(R) .
\end{gathered}
$$

Substitution shows that equations (1)-(3) are automatically satisfied. The condition under which equation (4) is satisfied is found to be a generalization of equation (22):

$$
f=\frac{12 a(3-a)-24\left(\alpha_{T} / \alpha\right)(1-a) a}{(a+5)(3-a)^{2}}, \text { and } \epsilon=0 .
$$

The first condition gives a relation between $f$ and $a$.

In Figure 8 we show global solutions with $f=0.5$ without turbulent energy transport $\left(\alpha_{T}=0, a=0.565\right)$, and we compare these with the analytical solution given above. Once again, the analytical solution fits $c_{\mathrm{s}}$ very well for all $R$ and fits $\rho$ and $l$ well in the outer regions. As before, the analytical solutions for $\rho$ and $l$ deviate significantly from the numerical results in the inner regions.

\section{SUMMARY AND DISCUSSION}

In this paper, we identified three "connection conditions" which ADAF models should satisfy in order to be able to connect to standard thin disks: (1) the temperature and velocity of the gas should change by orders of magnitude in the transition region between the ADAF and the thin disk, (2) there should be super-Keplerian rotation in the transition region, and (3) there should be an outward energy flux. We derived a general form of the outer boundary conditions that global ADAF models must satisfy in order to be consistent with the three connection conditions. With these boundary conditions, we calculated numerical global ADAF models.

The interior regions of our global solutions show the same dependences on the $\alpha$ viscosity parameter as those described in the literature (e.g., Narayan et al. 1997b), namely, we find lower angular momentum and lower density of the gas for larger values of $\alpha$ (Fig. 1). At the outer boundary, however, we find that all physical quantities except $v$ and $\rho$ are independent of $\alpha$. We also examined the effect of turbulent energy transport on the global structure of ADAFs and found little effect, especially in the case of $\epsilon=0$ (Fig. 2). In a sense, the presence of the energy flux due to turbulence is hidden in the flow (since the divergence of the flux is close to zero), until it reaches the outer transition zone. A small deviation from $4 \pi R H F_{\text {turb }}=$ constant is, however, enough to produce a significant effect on the Bernoulli parameter $b$ of the flow (Fig. 3).

In all our global models we find the Bernoulli parameter to be positive for a wide range of radii even though it is negative at the outer boundary and zero at the horizon (Fig. 3 ). Even in the case of $\epsilon=0$, where analytical work suggests that $b$ should be either zero (NY) or negative except for a narrow inner region in the case of a large $\alpha_{T}(=\alpha)$ (Nakamura 1998), we find a positive value of $b$ over a range of radii; however, the value of $b$ is not very large. In the case of $\epsilon=1, b$ is significantly larger and approaches the value derived by Narayan \& Yi (1994) from their self-similar solution, when $\alpha_{T}=0$. Turbulent energy transport enhances the value of $b$ still further by pumping out energy from the innermost region of the flow to the outside, although the value is below that derived from the self-similar solution for $\alpha_{T}=\alpha$. The effect is particularly noticeable when $\epsilon=1$ and $R_{\mathrm{tr}}$ is large. We suggest that under these conditions ADAFs may have particularly powerful outflows (Narayan \& Yi 1994, 1995a; Blandford \& Begelman 1998).

In this paper we have followed NY and Honma (1996) and assumed that the turbulent energy flux is proportional to (the negative of) the entropy gradient. This is similar to the prescription that is used to model convective heat transport in stellar interiors. Sometimes in the literature, e.g., Gruzinov (1999), the turbulent energy flux is assumed to be proportional to the temperature gradient (as for microscopic thermal conduction). This is not easy to justify for turbulent transport in a compressible medium since a simple mixinglength theory of turbulent convection indicates that thermal energy is transported down the entropy gradient.

One of the main motivations of this paper was to compare numerical global ADAF solutions with two analytical solutions in the literature, namely those of Honma (1996) and NY. The comparisons are shown in Figures 4-8.

Honma's solution provides an excellent approximation for the sound speed $c_{\mathrm{s}}$ over the whole range of $R$ but fits the density $\rho$ and the specific angular momentum $l$ only near the outer boundary. In contrast, NY's self-similar solution is invalid near the outer boundary but gives a reasonable
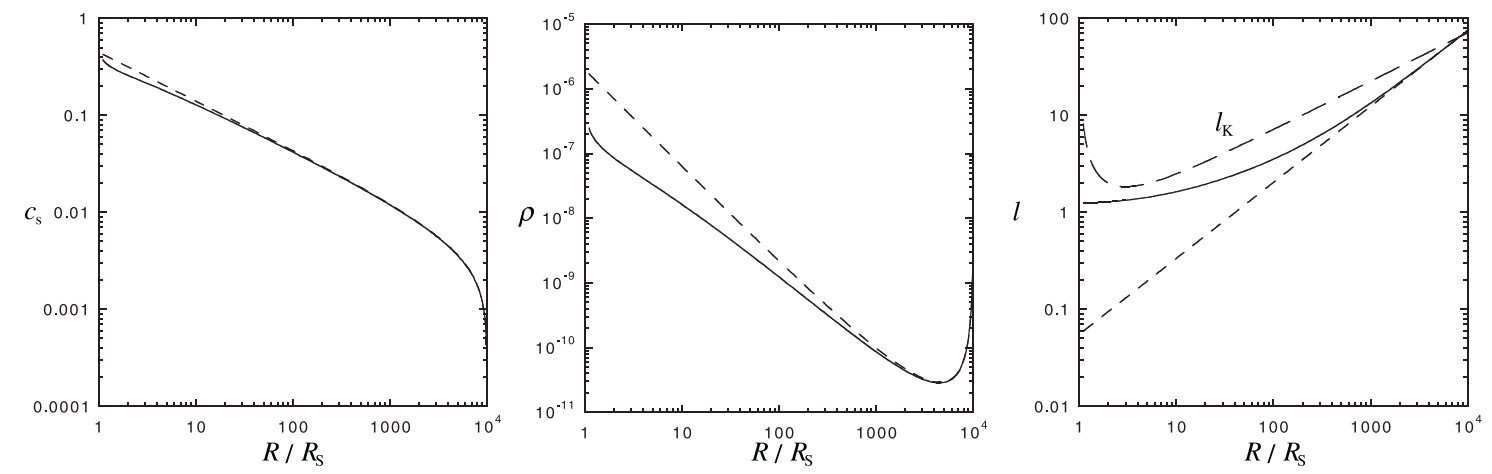

FIG. 8.-Comparison of the numerical global solution with an extension of Honma's analytical solution described in the paper. The case shown corresponds to $\epsilon=0, \alpha=0.1, \alpha_{T}=0, f=0.5, a=0.565, R_{\mathrm{tr}}=10^{4} R_{\mathrm{S}}$. Left panel: Isothermal sound speed $c_{\mathrm{s}}$. Center panel: Density $\rho$ (for $\dot{m}=0.01$ ). Right panel: Specific angular momentum $l$. Solid lines correspond to the numerical solution, and dashed lines to the analytical solution. The long-dashed line in the right panel represents the Keplerian angular momentum. 
approximation for the global solution at intermediate radii, especially when $\epsilon=1$. Thus, Honma's solution and NY's self-similar solution are complementary to each other; the former is good in the transition region near the outer boundary and the latter is better away from the boundary.

Unfortunately, neither analytical solution is very good as we approach the sonic radius near the black hole, and we have not succeeded in deriving a third analytical approximation to represent this region of the flow. The lack of a reliable approximation for the inner regions of the flow is particularly serious when $R_{\mathrm{tr}}$ is small, say $R_{\mathrm{tr}} \sim 100 R_{\mathrm{s}}$. In this case, neither of our two analytical forms fits the global solution well except in the region very close to the outer boundary, where Honma's solution is barely a good approximation.

As part of our analysis, we have extended Honma's solution to include the effect of partial cooling $(f<1)$ in the case of $\epsilon=0$. Once again, the extended solution accurately represents the physical quantities near the outer boundary. We thus conclude that Honma's solution represents well the structure of the transition region of ADAFs for a variety of conditions.

We would like to emphasize that Honma's solution and NY's self-similar solution are distinct in the sense that Honma's solution cannot be expressed as the limiting case of the self-similar solution or vice versa. This is most easily seen by considering the slopes of the angular momentum profiles in Figure 6. The self-similar solution has $d \ln \Omega /$ $d \ln R=-3 / 2$, while Honma's solution has $d \ln \Omega /$ $d \ln R=-1$, and this does not change even if the transition radius tends to infinity.

Given that there are two distinct analytical solutions, which one does nature choose? The answer perhaps depends on the outer boundary condition. If the mass to an ADAF is supplied by a wind, as in high-mass X-ray binaries or the Galactic center source Sagittarius $A^{*}$, or from a quasi-spherical Bondi-like inflow, as in cooling flows in the nuclei of elliptical galaxies, the gas may make a smooth transition directly into something resembling the NY selfsimilar solution. On the other hand, if the mass is supplied from a standard disk, as in Roche-lobe overflow fed X-ray binaries or some LINER galaxies like NGC 4258, the transition is likely to be represented by Honma's solution, at least in the transition region. Even in this case, the selfsimilar solution might be a better representation of the flow inside the transition region.

Last, we should emphasize that although we have constructed ADAF models that satisfy the necessary "connection conditions," our models are still incomplete. We do not determine the value of the transition radius, for instance. For this we need to consider the detailed thermal structure of the transition region, with a full treatment of radiative cooling. When such a calculation is done selfconsistently, the connection between the ADAF solution we have calculated and the outer disk will presumably occur over a narrow transition region where the angular velocity would decrease from the super-Keplerian rotation of the ADAF to the Keplerian rotation of the outer disk. Conceivably, the specific angular momentum may decrease with increasing radius in this layer (Honma 1996), in which case the transition region would violate the Rayleigh stability criterion and might be unstable. (A careful discussion is, however, necessary to judge whether the instability really occurs, since in the region where the specific angular momentum decreases with increasing radius the effective gravity is directed outward, and the convectively stable density stratification would strongly resist the instability.) Inside the super-Keplerian zone, there is a zone where the density increases outward even though the rotation is subKeplerian. In this region we may expect Rayleigh-Taylorlike instabilities. We feel that the instability may have interesting consequences. For instance, the instability could be the source of the strong time variability seen in the hard state of X-ray binaries (see Manmoto et al. 1997). It may also provide the strong turbulent energy transport that is needed in the transition layer in order to cause the cold gas in the disk to evaporate into the ADAF (Honma 1996).

T. M. and S. K. thank Fumio Honma for providing the basic part of the numerical code used in this work, and $\mathrm{T}$. M. thanks Ann Esin for discussions and numerous useful comments. This research was supported in part by the National Science Foundation under grant PHY 94-07194 and NASA under grant NAG 5-2837. T. M. is supported by Research Fellowships of the Japan Society for the Promotion of Science for Young Scientists.

\section{APPENDIX A}

\section{SOLUTIONS NEAR THE BOUNDARY AND ENERGY FLUX THROUGH THE BOUNDARY}

Let us introduce dimensionless variables $\tilde{f}, g, \tilde{h}$ by

$$
\begin{gathered}
\tilde{f}=\frac{c_{\mathrm{s}}^{2}}{v_{\mathrm{K}}^{2}}, \\
g=-\left(\frac{d \ln \Omega}{d \ln R}\right)^{-1}, \\
\tilde{h}=\frac{\Omega^{2}}{\Omega_{\mathrm{K}}^{2}} .
\end{gathered}
$$

We consider first the case when $\alpha_{T}=0$. The equation of radial force balance, and the equations describing the conservation of angular momentum and thermal energy then take the forms (Honma 1996)

$$
\frac{\alpha^{2} \tilde{f}^{2}}{2 g^{2}}\left(1-2 \frac{d \ln \tilde{f}}{d \ln x}\right)+\frac{5}{2} \tilde{f}+\frac{1}{2} x \frac{d \tilde{f}}{d x}-\frac{d \ln g}{d \ln x} \tilde{f}-1+\tilde{h}=0,
$$




$$
\begin{gathered}
\frac{2}{g}=3-\frac{d \ln \tilde{h}}{d \ln x}, \\
-\frac{3}{2}(\epsilon+2) \frac{d \tilde{f}}{d x}+\frac{3}{2} \epsilon \tilde{f}+\frac{d \ln g}{d \ln x}=\frac{\tilde{h}}{g} f .
\end{gathered}
$$

Following the text, we expand $\tilde{f}$ around $x=1$ as

$$
\tilde{f}=f_{0}(1-x)+f_{1}(1-x)^{2}+\cdots .
$$

Since $g$ and $\tilde{h}$ have finite values at $x=1$, we expand them as

$$
\begin{aligned}
& g=g_{0}+g_{1}(1-x)+\cdots, \\
& \tilde{h}=h_{0}+h_{1}(1-x)+\cdots .
\end{aligned}
$$

Equating the zeroth- and first-order terms in $(1-x)$ in equation (A4), respectively, we have

$$
\begin{gathered}
\frac{1}{2} f_{0}=1-h_{0}, \\
3 f_{0}+f_{1}-\frac{g_{1}}{g_{0}} f_{0}=-h_{1} .
\end{gathered}
$$

Similarly, the zeroth-order terms of equation (A5) give

$$
\frac{2}{g_{0}}=3-\frac{h_{1}}{h_{0}}
$$

and the zeroth- and first-order terms in equation (A6) give

$$
\begin{gathered}
-\frac{3}{2}(\epsilon+2) f_{0}=\frac{h_{0}}{g_{0}} f \\
-\frac{3}{2}(\epsilon+2)\left(2 f_{1}+f_{0}\right)+\frac{3}{2} \epsilon f_{0}+f_{0} \frac{g_{1}}{g_{0}}=\frac{h_{0}}{g_{0}}\left(\frac{h_{1}}{h_{0}}-\frac{g_{1}}{g_{0}}\right) f .
\end{gathered}
$$

The above five equations are simultaneous algebraic equations for six coefficients $\left(f_{0}, f_{1}, g_{0}, g_{1}, h_{0}\right.$, and $\left.h_{1}\right)$. They can be solved with one free parameter, say $g_{0}$. Results are shown in the text, where $g_{0}$ is written simply as $g$. Coefficients of higher order terms in $(1-x)$ of $\tilde{f}, g$, and $\tilde{h}$ may be derived by expanding equations (A4)-(A6) to higher powers with respect to (1 - $x$ ).

In the case of $\alpha_{T} \neq 0$, the order of the differential equation that describes the energy equation increases. Corresponding to this, when we carry out expansions of the basic equations with respect to $(1-x)$, two free parameters, say $g_{0}$ and $g_{1}$, appear. Except for this, the procedure is the same as above and is straightforward. The results are not given here, however, since the expressions are somewhat lengthy. The two free parameters are, of course, determined uniquely when a global solution is obtained.

The total energy flux, $W$, carried outward in the radial direction through a cylindrical surface at radius $R$ is generally written as

$$
W=\dot{M}\left(\frac{1}{2} v^{2}+\frac{1}{2} \Omega^{2} R^{2}-\psi+\frac{\gamma}{\gamma-1} c_{\mathrm{s}}^{2}\right)+4 \pi R H F_{\mathrm{turb}}-4 \pi R^{3} \alpha \rho c_{\mathrm{s}} H^{2} \frac{d \Omega}{d R} \Omega .
$$

For simplicity, we consider again the case of $\alpha_{T}=0$. Then, by using the dimensionless variables introduced above, we have

$$
W=-\frac{1}{2} \dot{M} c^{2} \frac{1}{x}\left[\frac{\alpha^{2}}{2} \frac{\tilde{f}^{2}}{g^{2}}-1-\frac{1}{2} \tilde{h}+\frac{3}{4}(\epsilon+2)\right] \text {. }
$$

In the lowest order of expansion with respect to $(1-x)$, we have at $x=1$

$$
W=\frac{1}{2} \dot{M} c^{2}\left(1+\frac{1}{2} h_{0}\right),
$$

which gives the expression given in the text. The next term in the expansion vanishes identically, as is to be expected by the constancy of the flux.

In the case of $\alpha_{T} \neq 0$, the expression for the energy flux through the boundary is more complicated and is not given. However, the flux is certainly directed outward, since the turbulent heat flux is always outward.

\section{REFERENCES}

Abramowicz, M. A., Chen, X., Kato, S., Lasota, J.-P., \& Regev, O. 1995, ApJ, 438, L37

Abramowicz, M. A., Czerny, B., Lasota, J.-P., \& Szuszkiewicz, E. 1988, ApJ, 332, 646

Abramowicz, M. A., Igumenshchev, I. V., \& Lasota, J.-P. 1998, MNRAS, 479,179
Abramowicz, M. A., Lasota, J.-P., \& Igumenshchev, I. V. 1999, ApJ, in press

Blandford, R. D., \& Begelman M. C. 1998, MNRAS, 303, L1

Chen, X., Abramowicz, M. A., \& Lasota, J.-P. 1997, ApJ, 476, 61

Chen, X., Abramowicz, M. A., Lasota, J.-P., Narayan, R., \& Yi, I. 1995, ApJ, 443, L61 
DiMattio, T., \& Fabian, A. C. 1997, MNRAS, 286, L50

DiMatteo, T., Fabian, A. C., Rees, M. J., Carrilli, C. L., \& Ivison, R. J. 1999, MNRAS, 305, 492

Esin, A. A., Narayan, R., Cui, W., Grove, J. E., \& Zhang, S. N. 1998, ApJ, 505,854

Fabian, A. C., \& Rees, M. J. 1995, MNRAS, 277, L55

Gammie, C. F., Narayan, R., \& Blandford, R. D. 1999, ApJ, 516, 177

Gruzinov, A. 1999, preprint (astro-ph/9809265)

Hameury, J.-M., Lasota, J.-P., McClintock, J. E., \& Narayan, R. 1997, ApJ, 489,234

Honma, F. 1996, PASJ, 48, 77

Ichimaru, S. 1977, ApJ, 214, 840

Kato, S., \& Nakamura, K. E. 1998, PASJ, 50, 559

Kato, S., Yamasaki, T, Abramowicz, M. A., \& Chen, X. 1997, PASJ, 49, 221

Lasota, J.-P., Abramowicz, M. A., Chen, X., Krolik, J., Narayan, R., \&Yi, I. 1996, ApJ, 462, L142

Mahadevan, R. 1997, ApJ, 477, 585 1998, Nature, 394, 651

Manmoto, T., Mineshige, S., \& Kusunose, M. 1997, ApJ, 489, 791

Manmoto, T., Takeuchi, M., Mineshige, S., Matsumoto, R., \& Negoro, H. 1996, ApJ, 464, L135

Meyer F., \& Meyer-Hofmeister E. 1994, A\&A, 288, 175
Nakamura, K. E. 1998, PASJ, 50, L11

Nakamura, K. E., Matsumoto, R., Kusunose, M., \& Kato, S. 1997, PASJ, 49,503

Narayan, R. 1996, ApJ, 462, 136

Narayan, R., Barret, D., \& McClintock, J. E. 1997a, ApJ, 482, 448

Narayan, R., Kato, S., \& Honma, F. 1997b, ApJ, 476, 49

Narayan, R., Mahadevan, R., Grindlay, J. E., Popham, R. G., \& Gammie, C. 1998, ApJ, 492, 554

Narayan, R., McClintock, J. E., \& Yi, I. 1996, ApJ, 457, 821

Narayan, R., \& Yi, I. 1994, ApJ, 428, L13 (NY) 1995a, ApJ, 444, 231

1995b, ApJ, 452, 710

Narayan, R., \& Yi, I., \& Mahadevan, R. 1995, Nature, 374, 623

Paczyński, B., \& Wiita, P. J. 1980, A\&A, 88, 23

Rees, M. J., Begelman, M. C., Blandford, R. D., \& Phinny E. S. 1982, Nature, 295,17

Reynolds, C. S., DiMatteo, T., Fabian, A. C., Hwang, U., \& Canizares, C. R. 1997, MNRAS, 283, L111

Shakura, N. I., \& Sunyaev, R. A. 1973, A\&A, 24, 337

Shapiro, S. J., Lightman, A. P., \& Eardley, D. M. 1976, ApJ, 204, 187

Wu, X. 1997, MNRAS, 292, 113 